\title{
The Mammary Gland and the Homeobox Gene Otx1
}

\author{
Ilaria S. Pagani, $\mathrm{PhD}, *$ Alessandro Terrinoni, $\mathrm{PhD},{ }^{\dagger}$ Laura Marenghi, $\mathrm{PhD}, *$ \\ Ileana Zucchi, PhD ${ }^{\ddagger}$ Anna M. Chiaravalli, PhD,${ }^{\mathbb{S}}$ Valeria Serra, $\mathrm{PhD},{ }^{\dagger}$ \\ Francesca Rovera, MD, "Silvia Sirchia, PhD,** Gianlorenzo Dionigi, MD, ${ }^{\dagger \dagger}$ \\ Monica Mozzo, MD,** Annalisa Frattini, PhD, ${ }^{*}$ Alberta Ferrari, MD, \\ Carlo Capella, MD, $\stackrel{\mathbb{S}}{\mathbb{S}}$ Francesco Pasquali, MD, ${ }^{*}$ Francesco L. Curto, PhD,* \\ Alberto Albertini, MD ${ }^{*}$ Gerry Melino, MD, ${ }^{\dagger}$, and Giovanni Porta, MD*
}

*Department of Experimental and Clinical Biomedical Sciences, University of Insubria, Varese, Italy; ${ }^{\dagger}$ Biochemistry Laboratory, IDI-IRCCS, C/O Department of Experimental Medicine and Biochemical Sciences, University of Rome "Tor Vergata", Rome, Italy; Institute of Biomedical Technologies, National Research Council, Milan, Italy; ${ }^{\mathbb{S}}$ Department of Pathology, Ospedale di Circolo, Varese, Italy; "Department of Surgical Sciences, University of Insubria, Varese, Italy; * Department of Medicine, Surgery and Dentistry, Medical Genetics Unit, Universita' degli Studi di Milano, Milano, Italy; ${ }^{\dagger \dagger}$ Endocrine Surgery Research Center, Department of Surgical Sciences, University of Insubria, Varese, Italy; ${ }^{\star}$ Fondazione I.R.C.C.S. Policlinico San Matteo, Pavia, Italy; ${ }^{\mathbb{S}}$ Department of Human Morphology, Anatomic Pathology Unit, Centro Insubre di Biotecnologie per la Salute Umana, Ospedale di Circolo, University of Insubria, Varese, Italy; and ${ }^{\top \uparrow}$ Medical Research Council, Toxicology Unit, Leicester University, Leicester, United Kingdom

Abstract: The mammary gland, the unique organ that primarily form at puberty, is an ideal model to study the functions of homeobox (HB) genes in both development and tumorigenesis. HB genes comprise a large family of developmental regulators that have a critical role in cell growth and differentiation. In the normal mammary gland, homeobox genes are involved in ductal formation, epithelial branching, and lobulo-alveolar development by regulating epithelial proliferation and differentiation. The HB genes are controlled in a spatial and temporal manner in both stromal and epithelial cells. They are coordinately regulated by hormones and extracellular matrix, suggesting that many signaling pathways are involved in homeobox gene functions. When homeobox genes are misexpressed in animal models, different defects are displayed in mammary gland development. Aberrant expression of homeobox genes, overexpressed or downregulated, is found in primary carcinomas and in breast cancer. The Otx1 HB gene is a classic regulatory of nervous system development during embryogenesis. Postnatally Otx1 is transcribed in the anterior pituitary gland, where activates transcription of the pituitary hormones, and plays a role in hematopoiesis, enhancing pluripotent cells, and erythroid differentiation. Otx1 can still be detected in mature cells of the erythroid and megacaryocytic lineage. During cyclical development of mammary gland, the Otx1 gene is overexpressed in lactation, confirming a role of this transcription factor in cell differentiation. Recent studies report that Otx1 is overexpressed in breast cancer. Otx1 is expressed during embryogenesis, and it is expressed again during carcinogenesis, implying its possible function in differentiation of neoplastic cells.

Key Words: breast cancer, homeobox genes, lactation, mammary gland, Otx1

$\mathrm{T}$ he mammary gland, unlike most mammalian organs, which develop embryonically with linear progression until the maturity, is the unique organ that primarily forms at puberty. Development of the mammary gland may be divided into both a linear

Address correspondence and reprint requests to: llaria S. Pagani, PhD and Giovanni Porta, MD, Department of Experimental and Clinical Biomedical Sciences, University of Insubria, via Dunant 5, 21100 Varese, Italy, or e-mails: ilaria.pagani@uninsubria.it, giovanni.porta@uninsubria.it.

DOI: 10.1111/j.1524-4741.2010.01006.x

(c) 2010 Wiley Periodicals, Inc., 1075-122X/10

The Breast Journal, Volume 16 Suppl. 1, 2010 S53-S56 and cyclical phase, induced by hormonal changes. Puberty is preceded by several hormonal changes in the body: the female releases follicle-stimulating hormone (FSH) and lutenizing hormone ( $\mathrm{LH})$ in a cyclic pattern, from the anterior pituitary gland. These hormones stimulate the ovaries to synthesize and release female sex steroid hormones, estrogens and progestins, which induce waves of proliferation in the ducts $(1,2)$. During the puberty, ductal outgrowth rapidly increases under hormonal stimulation, resulting in side branching. The final differentiation stage is 
achieved during pregnancy and lactation, when numerous lobulo-acinar structures containing the milk-secreting alveolar cells are formed through extensive proliferation, followed by terminal differentiation. At the end of lactation, the weaning is accompanied by massive apoptosis and tissue remodeling, and the gland reverts to a structure resembling that before pregnancy. Different pathways, which regulate cellular growth, differentiation, and apoptosis, are implicated in pregnancy, lactation, and involution. These pathways involve stromal-epithelial interactions and are modulated by circulating hormones, local growth factors, cellular-extracellular matrix interactions, and by cell-cell interactions, which lead to remodeling of epithelial cell types (3).

The proliferation and the differentiation of mammary epithelial cells (MECs) is supported by the presence of multipotent mammary stem cells (MASCs) (4) in the "terminal end bud", the structures where ductal elongation and branching take place (1). Stem cells (SCs) are cells with a capacity to self-renew and to generate daughter cells, which can differentiate into multiple cell type that are found in the mature tissues. These two functions can be accomplished through a single asymmetric self-renewing mitotic division, in which one progeny retains SC identity and the other undergoes multiple rounds of divisions before entering a postmitotic fully differentiated state (5). The cells that form the intermediates between SCs and terminally differentiated cells are usually referred to as progenitor cells, transit cells, and transit amplifying cells (6). The asymmetric division is a particularly attractive strategy, which the SCs can use to perpetuate themselves and generate differentiated progeny, maintaining an appropriate number of cells. SCs can also use symmetric division, when they are expanding in number during development or after injury, to generate two daughter cells that are destined to acquire the same fate, to become SCs or differentiated cells. These two strategies can be used together or separately to produce an appropriate number of SCs and/or differentiated daughters. This balance is controlled by developmental and environmental signals coming from the "SC niche". The microenvironment is composed of myoepithelial and endothelial cells, fibroblasts, myofibroblasts, leukocytes, other cell types, and extracellular matrix molecules, which support SCs functions (5). In the mammary gland, the SC niche modulates the tissue specificity of the normal breast, such as the growth, survival and polarity, and the role of MASCs. A recent work by Villadsen identifies a candidate SC niche in the ducts and zones containing progenitors cells in lobules. Putative MASCs residing in ducts are essentially quiescent, whereas the progenitor cells in the lobules are more likely to be actively dividing (7). SCs can be divided in two functional classes: cells responsible for tissue renewal and cells that are inactive until required in response to environmental factors.

The microenvironment influences both normal breast and breast cancer cells development (8). The breast cancer is the leading cause of cancer-related death in women. The natural history of breast cancer, involves progression through defined pathological and clinical stages, starting with ductal hyperproliferation, with subsequent evolution into in situ and invasive carcinomas, and finally into metastatic disease (9). The earliest stage seems to depend on the ability of transformed epithelial cells to recruit and then subvert a variety of stromal cells originating from adjacent normal tissue (8). The initiation of breast cancer is because of transforming events, genetic and epigenetic, in a single cell, a breast cancer stem cell (BCSC), and subsequent tumor progression is driven by the accumulation of additional genetic changes combined with clonal expansion and selection (9). According to "the cancer stem cell (CSC) hypothesis", the tumors are derived from mutated SCs that have retained, or progenitors that have regained, the SC property of cell self-renewal (10). The CSCs, with symmetric divisions, produce differentiated cancer cells unable to undergo self-renewing cell division. Thus, only CSCs can accumulate additional genetic changes that can drive tumor progression and drug resistance. This model explains the intratumoral diversity of breast cancer, which is not a single disease, but is highly heterogeneous at both the molecular and clinical levels. Depending on molecular differences, breast cancer can be divided into five major molecular subtypes: basallike, luminal A, luminal $\mathrm{B}, \mathrm{HER} 2^{+} / \mathrm{ER}^{-}$, and normal breast-like (9).

Several studies demonstrate that a number of genes, such as homeobox genes, are mutated in breast cancer. The homeobox (HB) genes, discovered first in the fruit fly, Drosophila melanogaster, are a family of regulatory genes that encode transcription factors, which play a vital role in embryonic morphogenesis. Their functions are critical in specifying cell identity, in cell differentiation, and in positioning of bodily axis during embryo development. These genes contain a 
common 180-nucleotide sequence, termed "homeobox", that encodes a 60-aminoacid domain, the "homeodomain", responsible for recognition and binding of DNA-specific sequences on the target genes. This domain recognizes DNA sequences ranging from TGAT to TAAT to TTAT and TTAC (11). Many homeoproteins have been shown to function as transcriptional regulators, some as activators and others as repressor. Recent evidences indicate that $\mathrm{HB}$ genes are involved in the control of cell growth, through regulation of cell cycle, and when dysregulated are involved in tumorigenesis. The deregulated expression of $\mathrm{HB}$ genes has been described in many solid tumors, such as carcinoma, and in leukemias and lymphomas. It is widely accepted that many of the molecular pathways that underlie carcinogenesis represent aberrations of the normal process that control embryogenesis. The consequences of deregulated HB genes for carcinogenesis can be interpreted as an extension of their normal functions. The HB genes that show normal expression patterns in undifferentiated or proliferative cells are downregulated in differentiation and are re-expressed in cancers, such as breast cancer, whereas those that are normally expressed in differentiated tissues are downregulated in cancer progression (12).

The mammary gland, in which most maturation occurs postnatally, provides an ideal model for studying the functions of $\mathrm{HB}$ genes in both development and tumorigenesis. The expression of many HB genes has been detected in both normal mammary gland and neoplastic breast tissue. In the normal mammary gland, the expression of homeobox genes is coordinately regulated by hormones and extracellular matrix in a spatial and temporal manner in both stromal and epithelial cells. Animals with misexpressed HB genes display different extent of defects in ductal proliferation, side branching, and alveoli formation, implying that $\mathrm{HB}$ genes are important for normal mammary gland development (11). Recently, it has been demonstrated the expression of Otx1 gene, member of Otx family (Otx1, Otx2, Otx3, and Crx), homolog of the Drosophila orthodenticle (otd) gene, in normal and in tumoral breast tissue (A. Terrinoni et al., submitted). The Otx1 gene encodes transcription factors that bind with high affinity TAATCC/T DNA-specific sequence of target genes (13), which are involved in brain regionalization, corticogenesis, and organ sense development, during embryogenesis. Postnatally Otx 1 is also transcribed at relatively low levels in the anterior lobe of the pituitary gland, where activates transcription of the pituitary hormones (14). Otx1 also plays a role in hematopoiesis, enhancing SCs differentiation: it is expressed in bone marrow cells, especially in cells of the erythroid lineage, from progenitors and pluripotent cells to mature cells, and can still be detected in megakaryocytic lineage (15). Terrinoni et al. (submitted) have demonstrated that Otx1 gene is expressed during mouse mammary gland development and is overexpressed in lactation, confirming the role of this gene in the cell differentiation. Recently, it has been reported that, in humans, Otx1 is overexpressed specific subset of B cells in aggressive non-Hodgkin Lymphomas, in specific neural tumors, such as medulloblastomas of nodular/desmoplasmic subtype (16). Otx1 is also overexpressed in ductal and in lobular invasive breast cancer, and it has been detected in LA7 CSCs derived from rat mammary adenocarcinoma (A. Terrinoni et al., submitted).

In conclusion, the HB genes are involved in development of the mammary gland, by regulating epithelial proliferation and differentiation. The gain or loss of function of $\mathrm{HB}$ genes, with inappropriate effects on growth and differentiation, promotes tumorigenesis. The Otx1 gene plays a critical role during mammary gland remodeling by its overexpression during lactation. Recent studies show that Otx1 is overexpressed in ductal and in lobular invasive breast carcinoma, implying its possible function in differentiation of neoplastic cells.

\section{REFERENCES}

1. Richert MM, Schwertfeger KL, Ryder JW, Anderson SM. An atlas of mouse mammary gland development. J Mammary Gland Biol Neoplasia 2000;5:227-41.

2. Hovey RC, Trott JF, Vonderhaar BK. Establishing a framework for the functional mammary gland: from endocrinology to morphology. J Mammary Gland Biol Neoplasia 2002;7:17-37.

3. Dontu G, Al-Hajj M, Abdallah WM, Clarke MF, Wicha MS. Stem cells in normal breast development and breast cancer. Cell Prolif 2003;36:59-72.

4. Dontu G, Abdallah WM, Foley JM, et al. In vitro propagation and transcriptional profiling of human mammary stem/progenitor cells. Genes Dev 2003;17:1253-70.

5. Morrison SJ, Kimble J. Asymmetric and symmetric stem-cell divisions in development and cancer. Nature 2006;441:1068-74.

6. Smalley M, Ashworth A. Stem cells and breast cancer: a field in transit. Nature 2003;3:832-44.

7. Villadsen R, Fridriksdottir AJ, Ronnov-Jessen L, et al. Evidence for a stem cell hierarchy in the adult human breast. $J$ Cell Biol 2007;177:87-101.

8. Elenbaas B, Weinberg RA. Heterotypic signaling between epithelial tumor cells and fibroblasts in carcinoma formation. Exp Cell Res 2001;264:169-84. 
9. Polyak K. Breast cancer: origins and evolution. J Clin Invest 2007;117:3155-63.

10. Zucchi I, Artigiano S, Bertalot G, et al. Distinct populations of tumor-initiating cells derived from a tumor generated by rat mammary cancer stem cells. Proc Natl Acad Sci U S A 2008;105: 16940-5.

11. Chen H, Sukumar S. Role of homeobox genes in normal mammary gland development and breast tumorigenesis. J Mammary Gland Biol Neoplasia 2003;8:159-75.

12. Abate-Shen C. Deregulated homeobox gene expression in cancer: cause or consequence? Nature 2002;2:777-85.

13. Klein WH, Li X. Function and evolution of Otx proteins. Biochem Biophys Res Commun 1999;258:229-33.
14. Acampora D, Postiglione MP, Avantaggiato V, Di Bonito $\mathrm{M}$, Simenone A. The role of Otx and Otp genes in brain dvelopment. Int J Dev Biol 2000;44:669-77.

15. Levantini E, Giorgetti A, Cerisoli F, et al. Unsuspected role of the brain morphogenetic gene Otx1 in hematopoiesis. Proc Natl Acad Sci U S A 2003;100:10299-303.

16. Omodei D, Acampora D, Russo F, et al. Expression of the brain transcription factor OTX1 occurs in a subset of normal germinal-center B cells and in aggressive Non-Hodgkin lymphoma. Am J Pathol 2009;175:2609-17. 